\title{
Comparative effectiveness of switching paroxetine formulation for treatment of major depressive disorder: an open-label multicenter study
}

This article was published in the following Dove Press journal: Neuropsychiatric Disease and Treatment

\author{
Tempei Otsubo' \\ Yoshinori Watanabe $e^{2,3}$ \\ Seiji Hongo 3 \\ Mikichika Inoue 4 \\ Kimiko Akimoto 4 \\ Ken Murakami ${ }^{5}$ \\ Ryutaro Takahashi ${ }^{6}$ \\ Toshiaki Kikuchi ${ }^{7}$ \\ 'Department of Psychiatry, Tokyo \\ Women's Medical University \\ Medical Center East, Tokyo, Japan; \\ ${ }^{2}$ Himorogi Psychiatric Institute, Tokyo, \\ Japan; ${ }^{3}$ Nanko Clinic of Psychiatry, \\ Shirakawa, Japan; ${ }^{4}$ Ikebukuro Internal \\ Medicine, Tokyo, Japan; ${ }^{5}$ Murakami \\ Hospital, Tokyo, Japan; ${ }^{6}$ Takahashi \\ Clinic, Tokyo, Japan; ${ }^{7}$ Department of \\ Neuropsychiatry, Keio University \\ School of Medicine, Tokyo, Japan
}

Aim: To assess the effectiveness and safety of switching the antidepressant formulation from immediate-release (IR) to controlled-release (CR) paroxetine in patients with major depressive disorder (MDD).

Patients and methods: A total of 113 outpatients with MDD diagnosed according to the Diagnostic and Statistical Manual of Mental Disorders, Fourth Edition, Text Revision, and treated with a stable dose of IR paroxetine for at least 6 months were enrolled. Patients were then switched to CR paroxetine for 8 weeks. Effectiveness was evaluated by scores on the Himorogi Self-Rating Depression/Anxiety Scales (HSDS/HSAS) and the Clinical Global Impression Severity (CGI-S). Safety was evaluated based on the reported adverse drug reactions (ADRs). Medication satisfaction and preference were assessed based on questionnaire responses using Likert-type scales.

Results: The overall patient HSDS/HSAS scores significantly improved after switching from IR to CR paroxetine $(P<0.001)$. Furthermore, CR paroxetine was superior to IR paroxetine $(P<0.001)$ according to the results of the CGI-S evaluation. ADRs were experienced by 14 (12.4\%) patients, including dry mouth, nausea/vomiting, somnolence/drowsiness, and wakefulness/arousal during sleep. Satisfaction and preference for paroxetine improved after switching to the $\mathrm{CR}$ formulation $(P<0.001$; chi-square test).

Conclusion: These results suggest that switching the treatment from IR to CR paroxetine could improve depressive symptoms and decrease ADRs. However, these results may have been caused by the psychological effect of drug switching. Hence, future studies with blinded evaluation methods are required to confirm and expand our findings.

Keywords: depression, controlled-release paroxetine, drug formulation, antidepressant, immediate-release

\section{Introduction}

Globally, more than 300 million people suffer from depression, a condition associated with poor social functioning and high mortality. ${ }^{1}$ Clinical practice guidelines recommend using selective serotonin reuptake inhibitors (SSRIs) as a first-choice pharmacological option when treating patients with major depressive disorder (MDD)., ${ }^{2,3}$ However, one-third of these patients do not achieve remission with the initial antidepressant. ${ }^{4}$ In patients who have terminated treatment because of ineffectiveness or intolerability, switching to another antidepressant is recommended.,6

As reported by Papakostas et al, ${ }^{7}$ switching from SSRIs to non-SSRIs (eg, serotonin noradrenaline reuptake inhibitors) has significant advantages for helping patients with SSRI-resistant depression to achieve remission with lesser number needed to treat when
Correspondence: Toshiaki Kikuch Department of Neuropsychiatry, Keio University School of Medicine, 35 Shinanomachi, Shinjuku,

Tokyo 160-8582, Japan

$\mathrm{Tel}+8 \mid 33353$ I2I I

Fax +81353790187

Email kikuchi.psychiatry@gmail.com (c)
hereby accept the Terms. Non-commercial uses of the work are permitted without any further permission from Dove Medical Press Limited, provided the work is properly attributed. For permission for commercial use of this work, please see paragraphs 4.2 and 5 of our Terms (https://www.dovepress.com/terms.php). 
compared with switching from one SSRI to another SSRI. However, although sequential antidepressant strategies have been assessed, the efficacy of switching drug formulation has not been completely evaluated. In a post hoc analysis evaluating a switch from extended-release venlafaxine to desvenlafaxine, the venlafaxine nonresponders exhibited improvement during the double-blinded period after changing to treatment with desvenlafaxine during the open-label period. ${ }^{8}$ Although the switch was well tolerated, these results could have been associated with the change in study design (ie, double-blind to open-label), indicating that the effect of altering drug formulation has not been completely evaluated thus far.

Paroxetine is an SSRI available in both controlled-release (CR) and immediate-release (IR) formulations. Lower incidence of SSRI-induced gastrointestinal symptoms during early stage of treatment is expected with CR than IR formulation; this is possibly because of a degradable polymeric matrix (GEOMATRIX ${ }^{\mathrm{TM}}$ ) that permits slow dissolution and gradual drug release..$^{9-12}$ In fact, Golden et $\mathrm{al}^{10}$ reported that patients with depression taking CR paroxetine had significantly less nausea during 1 week after initiation of treatment than those taking IR paroxetine (14\% vs 23\%). The bioequivalence between $\mathrm{CR}$ and IR paroxetine has already been demonstrated, ${ }^{13}$ and the clinical efficacy and safety of CR paroxetine for MDD treatment have been shown to be similar to those of escitalopram, which has the highest probability of inducing remission. ${ }^{14,15}$

However, although the effectiveness and safety of CR paroxetine have been established, sequential formulation change of paroxetine has not been sufficiently evaluated.

Thus, we conducted an open-label prospective study to evaluate the effectiveness and safety of switching from an IR to a CR paroxetine formulation among patients with MDD in a clinical setting.

\section{Patients and methods}

This single-arm, open-label, 8-week trial was conducted from June 2012 to October 2012 at five psychiatric hospitals and clinics in Tokyo and Fukushima, Japan. Outpatients aged $\geq 18$ years who met the Diagnostic and Statistical Manual of Mental Disorders, Fourth Edition, Text Revision, criteria for MDD or MDD with an anxiety disorder and treated with a stable dose of IR paroxetine for at least 24 weeks were included. The exclusion criteria were diagnosed convulsive disorders, malignancy, personality disorders, substance abuse, depressive state caused by organic brain damage, and severe disorders of the liver, kidney, or circulation. We also excluded the following patients: those who were nursing or pregnant; those who had a strong suicidal tendency; those who were receiving other intensive forms of therapy, including cognitive behavioral therapy, psychodynamic psychotherapy, and electroconvulsive therapy; and/or those who had doses of concomitant drugs (excluding CR paroxetine) modified within the preceding 8 weeks.

The trial protocol was approved by the institutional review board of Himorogi Psychiatric Institute (Tokyo, Japan). All patients provided written informed consent before inclusion and after receiving a full description of the study (trial registration: UMIN-CTR, UMIN000010950). The patients were instructed to switch from IR to CR paroxetine at clinical visits. CR formulation was administered to patients at doses clinically equivalent to their prior IR dose (ie, $10 \mathrm{mg}$ IR dose $=12.5 \mathrm{mg}$ CR dose). ${ }^{13}$ After initiating therapy with $\mathrm{CR}$ paroxetine, the dosage was fixed for 8 weeks. During treatment, other intensive forms of therapy were prohibited, including cognitive behavioral therapy, psychodynamic psychotherapy, and electroconvulsive therapy. Use of adjunctive sedative-hypnotics that had been taken at baseline was allowed, while the use of new additional drugs was prohibited.

The primary clinical assessments pertaining to the drugswitching strategy were conducted at baseline (initiation of CR treatment) and at week 8 (study end point). Effectiveness was evaluated based on the Himorogi Self-Rating Depression Scale (HSDS) and the Himorogi Self-Rating Anxiety Scale (HSAS), ${ }^{16,17}$ which are 10 -item self-rating questionnaires used to assess and monitor the severity of depression and anxiety (score range 0-39, with higher scores indicating more severe symptoms). Both scales were developed by the Himorogi Psychiatric Institute to make the evaluation of depression and anxiety symptoms easier and less timeconsuming for both patients and physicians. ${ }^{16,17}$ Research has shown a good correlation between the HSDS and the Japanese version of the Hamilton Rating Scale for Depression ${ }^{17,18}$ as well as between the HSAS and both Japanese versions of the Hamilton Anxiety Rating Scale ${ }^{19}$ and the Sheehan PatientRated Anxiety Scale. Cronbach's alpha coefficients for the HSDS and the HSAS demonstrate acceptable reliabilities of 0.85 (95\% confidence interval [CI], 0.82-0.88) and 0.87 (95\% CI, 0.85-0.90), respectively. ${ }^{16,17}$

The Clinical Global Impression - Severity (CGI-S) scale was used at baseline and at week 8 to record patient symptoms. ${ }^{20}$ The CGI-S describes a patient's overall clinical state as a "global impression" by the rater, and it correlates well with the established research drug efficacy scales (eg, Hamilton Rating Scale for Depression) across a wide range of psychiatric indications. ${ }^{21}$ 
Patient satisfaction and preference for CR paroxetine were assessed by graded patient questionnaires during interviews with the investigators. Satisfaction was rated as follows: very satisfied, moderately satisfied, slightly satisfied, neutral, slightly dissatisfied, moderately dissatisfied, and very dissatisfied. We calculated the satisfaction rate as the proportion of patients who were very, moderately, or slightly satisfied. Patient preference for CR paroxetine over the prior antidepressant was rated as follows: much better, moderately better, slightly better, similar, slightly worse, moderately worse, and much worse. ${ }^{11}$ Finally, the safety of $\mathrm{CR}$ paroxetine was evaluated based on the reported adverse drug reactions (ADRs) and common laboratory test results during the study period.

We used SPSS Version 17.0 (SPSS Inc., Chicago, IL, USA) for all statistical analyses. Continuous variables were reported as mean \pm standard deviation, and discrete variables were reported as numbers with percentages. The HSDS/HSAS total and subitem scores were analyzed using analysis of variance (ANOVA) and paired $t$-test, respectively. Two-tailed $P$-values of $<0.05$ were considered statistically significant. The CGI-S and medication satisfaction/preference questionnaire scores at week 8 were compared with those at baseline using a chi-square test.

\section{Results}

A total of 350 patients were screened, of whom 205 were eligible and enrolled in the study. Of these, 113 (55.1\%) patients completed the switching intervention with sufficient adherence (Figure 1). The baseline characteristics are presented in Table 1, and changes in the HSDS/HSAS scores from baseline to week 8 are summarized in Table 2. Most patients were diagnosed as having MDD without comorbidities $(68.1 \%, \mathrm{n}=77)$. The most frequent initial dose of CR paroxetine was $25 \mathrm{mg} /$ day (32.7\%), followed by 12.5 (27.4\%) and $50(26.5 \%) \mathrm{mg} /$ day.

The mean total HSDS scores were significantly reduced from 12.2 at baseline to 10.2 at week $8(P<0.001$; ANOVA). $\mathrm{CR}$ formulation was superior to IR formulation for sleep disturbance (HSDS item 1), hyposexuality (HSDS item 5), disinclination (HSDS item 8), and lower satisfaction (HSDS item 10) $(P<0.05)$ (Table 2). In addition, the distribution of HSDS severity categories $(8 \mathrm{~W})$ was significantly better than that in HSDS $(0 \mathrm{~W})(P<0.001$; chi-square test). Overall, 27 subjects $(23.9 \%)$ showed improvement of at least one grade category, while $78(69.0 \%)$ showed no category change and eight (7.1\%) got worse (data shown in Table S1).

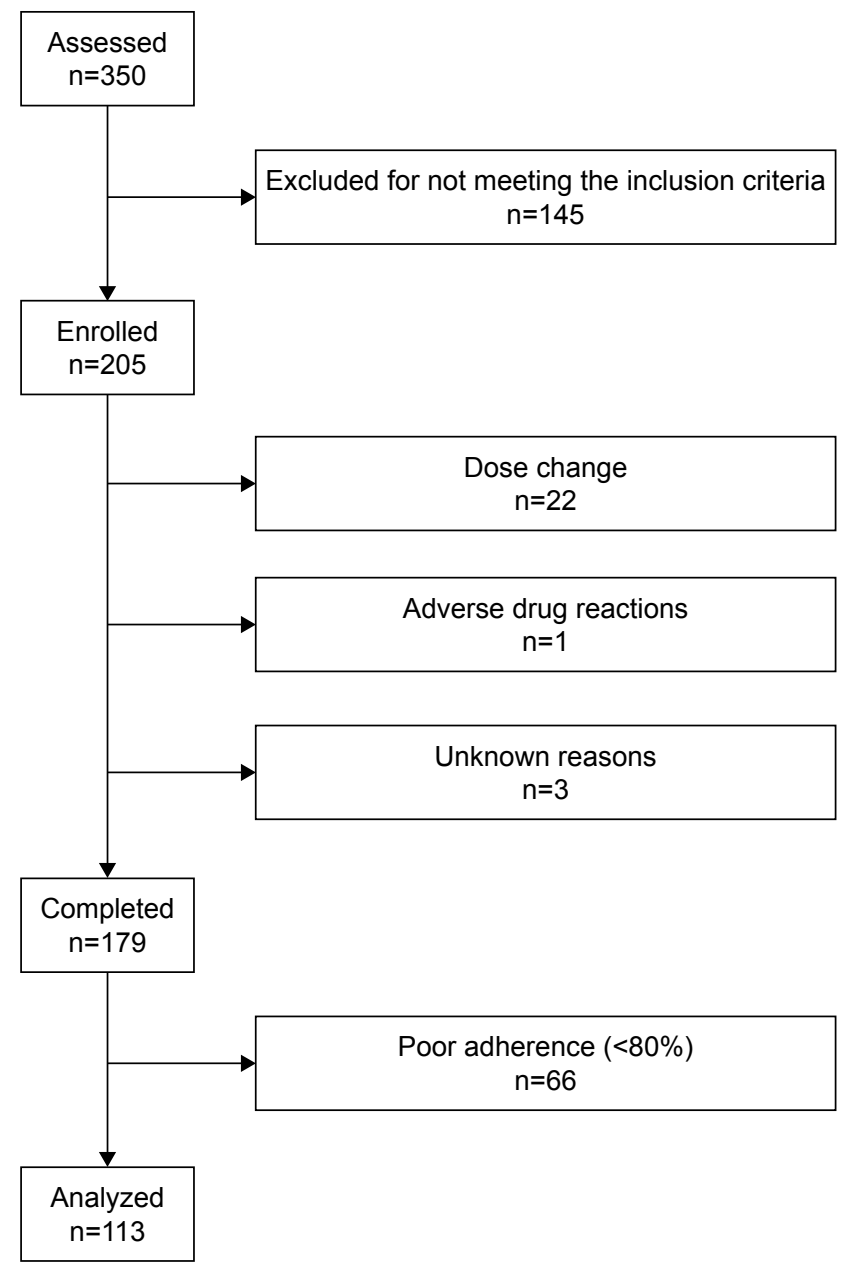

Figure I Study flow diagram.

Notes: The study flow diagram shows the patient distribution in this study. A total of 350 patients agreed to switch the medication from IR to CR. Of these, 205 patients were enrolled. During the study period, 22 patients changed the dose, one prematurely dropped out because of the onset of adverse events, and three quit without clear reasons. A total of 179 patients completed the 8-week study with stable doses of CR paroxetine. However, 66 patients were excluded from the analysis because of poor adherence ( $<80 \%$ of the theoretical total dosage).

Abbreviations: IR, immediate release; $C R$, controlled release.

The mean total HSAS scores were significantly reduced from 12.7 at baseline to 11.2 at week $8(P<0.001$; ANOVA). CR paroxetine was superior to IR paroxetine for the following subitem scores: autonomic nerve (HSAS item 3), stress (HSAS item 5), anticipatory anxiety (HSAS item 7), and sleeping (HSAS item 8) $(P<0.05)$ (Table 2).

Changes in the CGI-S scores are presented in Figure 2. The severity of patient symptoms was compared between baseline and week 8 , and there was a noticeable shift in favor of CR paroxetine, as reflected by CGI-S improvements at week $8(P<0.001$; chi-square test). Similarly, there were greater satisfaction and preference associated with CR paroxetine compared with IR paroxetine $(P<0.001$; chi-square 
Table I Baseline characteristics

\begin{tabular}{ll}
\hline Characteristics & $\mathbf{n}=\mathbf{I} \mathbf{l 3}$ \\
\hline Gender, female, $\mathrm{n}(\%)$ & $57(50.4)$ \\
Age, mean \pm SD (years) & $41.2 \pm \mathrm{I} 1.52$ \\
Disease duration, mean \pm SD (years) & $5.52 \pm 4.09$ \\
Diagnosis, $\mathrm{n}(\%)$ & \\
MDD & $77(68.1)$ \\
MDD with SAD & $22(19.5)$ \\
MDD with PD & $9(8.0)$ \\
MDD with OCD & $2(1.8)$ \\
MDD with other comorbidities & $3(2.7)$ \\
CR paroxetine initial dose, $\mathrm{n}(\%)$ & \\
I2.5 mg & $31(27.4)$ \\
$25 \mathrm{mg}$ & $37(32.7)$ \\
$37.5 \mathrm{mg}$ & $15(13.3)$ \\
$50 \mathrm{mg}$ & $30(26.5)$ \\
BZD dose, ${ }^{\mathrm{a}}$ mean \pm SD (mg/day) & $10.24 \pm 10.5$ \\
\hline
\end{tabular}

Note: ${ }^{2}$ Diazepam equivalent dosage.

Abbreviations: SD, standard deviation; MDD, major depressive disorder; SAD, social anxiety disorder; PD, panic disorder; OCD, obsessive-compulsive disorder; $\mathrm{CR}$, controlled release; BZD, benzodiazepine.

Table 2 Changes in depression/anxiety scores $(n=1 \mid 3)$

\begin{tabular}{|c|c|c|c|}
\hline Scale items & Baseline & Week 8 & $P$-value \\
\hline \multicolumn{4}{|l|}{ HSDS score, mean \pm SD } \\
\hline Total score & $12.22 \pm 8.16$ & $10.22 \pm 7.24$ & $<0.00 I^{* * *}$ \\
\hline I. Sleep disturbance & $2.11 \pm 2.40$ & $1.65 \pm 2.19$ & $0.015 I^{*}$ \\
\hline 2. Appetite & $0.65 \pm 1.12$ & $0.48 \pm 0.98 @$ & 0.161 \\
\hline 3. Anxiety & $1.98 \pm 1.70$ & $1.70 \pm 1.61$ & 0.052 \\
\hline 4. Fatigue & $1.36 \pm 0.94$ & $1.22 \pm 0.90$ & 0.095 \\
\hline 5. Hyposexuality & $1.30 \pm 0.99$ & $1.05 \pm 0.96$ & $<0.00 I^{* *}$ \\
\hline 6. Somatic disorder & $1.10 \pm 0.91$ & $1.00 \pm 0.88$ & 0.153 \\
\hline 7. Depression & $0.7 I \pm 0.73 @$ & $0.63 \pm 0.73$ & 0.183 \\
\hline 8. Disinclination & $1.27 \pm 0.90$ & $1.09 \pm 0.87$ & $0.008^{* *}$ \\
\hline 9. Diminished ability to think & $0.85 \pm 0.83$ & $0.73 \pm 0.74$ & 0.058 \\
\hline 10. Lower satisfaction & $0.87 \pm 0.88^{@}$ & $0.68 \pm 0.77$ & $0.002^{* *}$ \\
\hline \multicolumn{4}{|l|}{ HSAS score, mean \pm SD } \\
\hline Total score & $12.72 \pm 9.53$ & $1 \mathrm{I} .18 \pm 8.86$ & $<0.00 I^{* * *}$ \\
\hline I. Cardiovascular & $1.5 \mathrm{I} \pm 1.79$ & $1.29 \pm 1.62$ & 0.093 \\
\hline 2. Respiratory & $1.86 \pm 1.94$ & $1.68 \pm 1.86$ & 0.175 \\
\hline 3. Autonomic nerve & $2.7 I \pm 2.13$ & $2.42 \pm 1.92$ & $0.043 *$ \\
\hline 4. Gastrointestinal & I.13土1.00@ & $1.1 I \pm 1.00$ & 0.731 \\
\hline 5. Distress & $1.27 \pm 1.00^{\ddagger}$ & $\mathrm{I} .1 \mathrm{I} \pm 0.98$ & $0.016 *$ \\
\hline 6. Awful feeling & $0.66 \pm 0.93$ & $0.55 \pm 0.91$ & 0.074 \\
\hline 7. Anticipatory anxiety & $1.01 \pm 1.01$ & $0.82 \pm 0.90$ & $0.006^{* *}$ \\
\hline 8. Sleeping & $1.10 \pm 1.09$ & $0.88 \pm 1.01$ & $0.015^{*}$ \\
\hline 9. Depersonalization & $0.50 \pm 0.86$ & $0.42 \pm 0.79$ & 0.103 \\
\hline 10. Anxiousness & $1.00 \pm 0.97$ & $0.92 \pm 1.01 @$ & 0.304 \\
\hline
\end{tabular}

Notes: Evaluations were conducted at baseline, week 4, and week 8. Primary comparisons were made between baseline and week 8 scores. Data from week 4 were not included in the final analyses. ${ }^{@} \mathrm{n}=1 / 2$. Paired $t$-test was used for the subitem scores (week 8 vs baseline), and ANOVA for HSDS/HSAS total scores at baseline, week 4, and week 8. HSDS scores: 0-9, no depression; 10-15, mild depression; 16-20, moderate depression; 21-30, moderately severe depression; and $\geq 31$, severe depression. HSAS scores: 0-7, no anxiety; 8-15, mild anxiety; I6-20, moderate anxiety; $2 \mathrm{I}-30$, moderately severe anxiety; and $\geq 3 \mathrm{I}$, severe anxiety. ${ }^{*} \mathrm{n}=$ I I I. *Statistically significant at $\alpha=0.05$. **Statistically significant at $\alpha=0.01$.

Abbreviations: HSDS, Himorogi Self-Rating Depression Scale; SD, standard deviation; HSAS, Himorogi Self-Rating Anxiety Scale; ANOVA, analysis of variance.

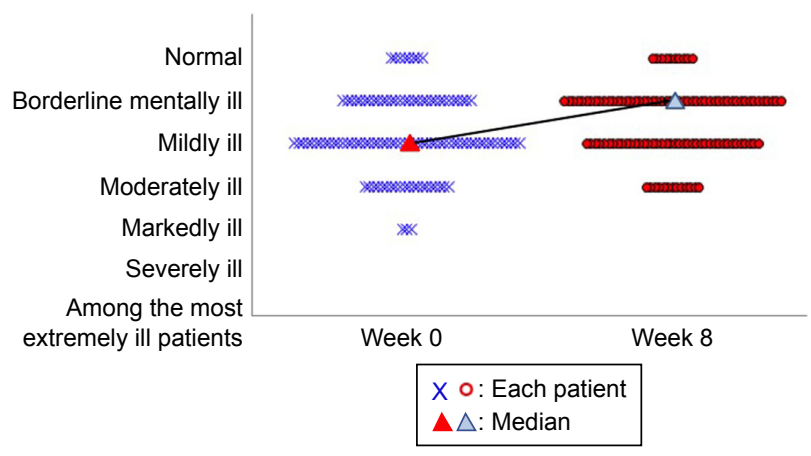

Figure 2 Clinical Global Impression - Severity in a dot-plot graph at baseline and at week 8 .

Notes: $X O$ indicate the patients and $\Delta \triangle P$ indicate median values at baseline and week 8 , respectively. The vertical axis shows the severity of patient symptoms. There was a significant improvement by week $8(P<0.00$ I; chi-square test), as shown by the change in medians from "mildly ill" for the immediate-release formulation to "borderline mentally ill" for the controlled-release formulation.

test; Figures 3 and 4), with $68.7 \%$ of patients reporting as being satisfied with $\mathrm{CR}$ paroxetine at week 8 .

ADRs were experienced by 14 (12.4\%) patients. The most common ADRs were dry mouth, nausea and/or vomiting, somnolence and/or drowsiness, and wakefulness and/or arousal during sleep ( $\mathrm{n}=2,14.3 \%$ each). Out of the 205 enrolled patients, only one $(0.49 \%)$ discontinued the treatment with CR paroxetine because of ADRs. Mean dosage of benzodiazepine was almost similar between baseline as well as IR period and 8 weeks after initiation of CR paroxetine (diazepam equivalent dosage was 10.2 and $10.1 \mathrm{mg} /$ day, respectively) in analyzed subjects.

\section{Discussion}

In the present study, we evaluated the effectiveness and safety of switching the paroxetine formulation from IR to CR for the treatment of MDD in 113 outpatients. In addition, patient

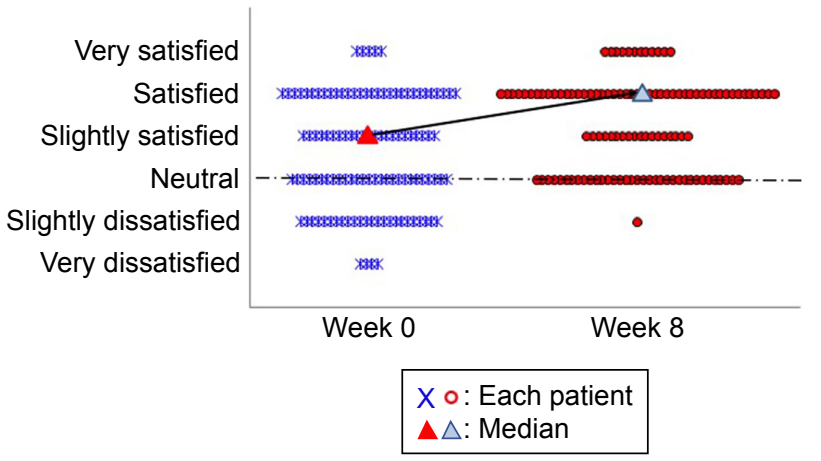

Figure 3 Satisfaction with paroxetine at baseline and week 8 in a dot-plot graph. Notes: The results are in response to the question "To what extent are you satisfied with your current medication"? X $\bigcirc$ indicate each patient and $\Delta \Delta$ indicate median values at baseline and week 8 , respectively. The vertical axis shows the degree of satisfaction. No patients were assessed as "very dissatisfied" after the change in treatment. There was a significant improvement by week $8(P<0.00$ I; chi-square test) as determined by the change in medians from "slightly satisfied" to "moderately satisfied". 


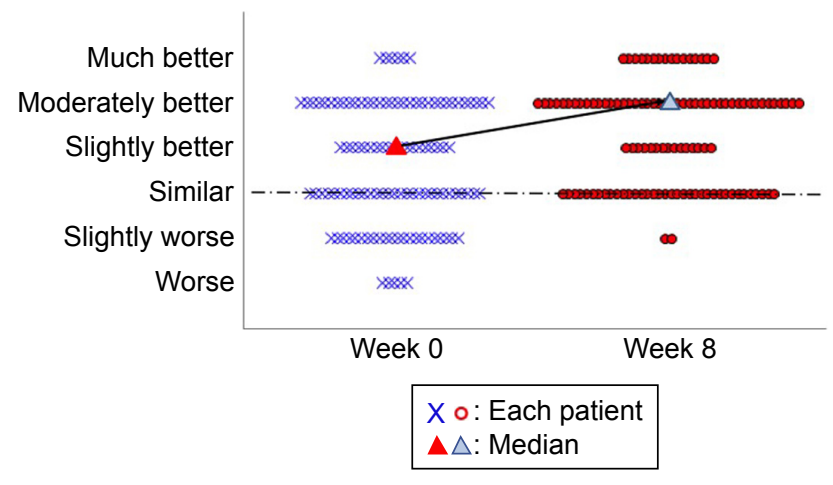

Figure 4 Preference for paroxetine at baseline and week 8 in a dot-plot graph. Notes: The data are in response to the question "Do you prefer the current medication to the previous one"? $X O$ indicate each patient and $\Delta \Delta \mathrm{n}$ indicate median values at baseline and week 8 , respectively. The vertical axis shows the degree of patient satisfaction. No patients were assessed as "much worse" after the treatment change. There was a significant improvement by week $8(P<0.00)$; chi-square test) as determined by the change in medians from "slightly better" to "moderately better".

satisfaction and preference for the formulation switch were also evaluated.

In clinical practice, if the first antidepressant is ineffective or the patient does not tolerate the drug, possible options include dosage escalation, introducing additional psychotropic drugs, or switching to another antidepressant. Each strategy has advantages and disadvantages. The patient's response to treatment can be partially facilitated by an increased dosage or augmentation strategies. Indeed, a recent report ${ }^{24}$ indicated that not only tricyclic antidepressants but also SSRIs exhibit a dose-response relationship, which is as high as $250 \mathrm{mg}$ for imipramine. Dosage escalation or use of adjunctive drugs may help to improve depressive symptoms; however, such therapies increase the risk of adverse events.

The effectiveness and safety of antidepressant-switching strategies have not been verified in comparison with continuing the initial antidepressant. ${ }^{27}$ However, it is true that switching to another antidepressant could help avoid drugdrug interactions, decrease polypharmacy, and prevent lower drug adherence. However, this approach could also lead to a loss of a partial response to the initial drug. This is further compounded by the fact that there is no solid evidence that switching antidepressants is more effective than continuing with the initial antidepressant. ${ }^{27}$ Further, although suggested target dose ranges exist when switching between or adding antidepressants, it is important to consider that there are no gold standard methods for judging dose equivalence when changing antidepressants. ${ }^{25,26}$

Given the risks associated with switching medication, changing only the drug formulation could be a safer method for managing the failure of the initial treatment. The results of the present study suggest that switching to a $\mathrm{CR}$ formulation of paroxetine can improve treatment effectiveness, while maintaining the partial response associated with the IR formulation. This may be because the change in formulation has less of an effect on the mechanism of action, and therefore maintains the drug's effectiveness. Although the effectiveness of switching to a CR formulation could not be confirmed in this single-arm open-label study, the results indicate that switching the drug formulation could be a safe option following an unsuccessful initial treatment of MDD.

The incidence of ADRs was $12.4 \%$ in this study, which is comparable to the results of a recent report regarding the safety and effectiveness of CR paroxetine in 3,213 Japanese patients with depression who switched from antidepressants of other classes (11.2\%). ${ }^{11}$ Further, the incidence of ADRs during IR paroxetine use has been reported as $22.4 \%$ (1,453 in 6,482 subjects) in similar post-marketing surveillance of patients with depression, panic disorder, obsessive-compulsive disorder, and social anxiety disorder in Japan; ${ }^{35}$ however, the survey for CR paroxetine only included depressive patients. The reported incidence of somnolence and nausea was also consistent with that reported in previous studies. ${ }^{9,11}$ Moreover, patient satisfaction at week 8 was good, which was also consistent with that of a post-marketing surveillance study of CR paroxetine (68.7\% vs $69.8 \%) .{ }^{11}$ As suggested by Kato et al, ${ }^{11}$ the reason for the low incidence of ADRs is the prolonged exposure to paroxetine (IR treatment for $\geq 6$ months) before switching to $\mathrm{CR}$ formulation. This is because several adverse reactions that cause the premature discontinuation of SSRIs tend to occur during the early stages of treatment. 28,29

Similarly, the safety and satisfaction results could have resulted from the properties of the $\mathrm{CR}$ formulation itself. In contrast to the IR formulation, CR paroxetine is gradually absorbed from the gastrointestinal tract, and therefore has less pronounced fluctuations in the peak and trough blood concentrations. Consequently, reduced ADRs and improved tolerability could have been related to the benefits of the CR formulation. Indeed, adherence to CR paroxetine in anxiety disorders has been reported to be higher than that of IR paroxetine.$^{30}$ Aljumah et al reported that patient satisfaction with antidepressants was positively correlated with their adherence $;{ }^{31}$ however, further investigation is required to confirm whether patient satisfaction with $\mathrm{CR}$ formulation can positively affect drug adherence and long-term treatment outcomes.

We revealed that switching from IR to CR formulation improved the satisfaction with antidepressants. However, it has been suggested that satisfaction or quality of life was affected by depressive states. ${ }^{32}$ In fact, Satisfaction with Study Medication 
Question at week 8 showed a statistically significant correlation with HSDS at week $8(r=-0.24, P<0.05)$. Therefore, the change of satisfaction with $\mathrm{CR}$ formulation may be caused secondarily by the improvement in depressive symptoms.

To evaluate the influence of the presence of comorbidity, we compared MDD subjects with comorbidity to those without comorbidity. The no-comorbidity group showed greater improvement in depressive and anxiety symptoms than the comorbidity group (HSDS sores: -2.32 vs -1.31 ; HSAS scores: -1.92 vs -1.44$)$, although the comparisons were not statistically significant $(P=0.32$ and $P=0.60$, respectively). This may be due to the lack of power of the statistical analysis (comorbidity group: $\mathrm{n}=77$; no-comorbidity group: $\mathrm{n}=36$ ); however, the result was consistent with those of previous reports that MDD subjects with comorbidity respond poorly to pharmacotherapy. ${ }^{33}$

\section{Limitations}

The current study has several limitations: 1) We did not include a control group, and the inclusion of either a control group or a crossover study design could have allowed for a more in-depth analysis of drug effectiveness and safety (eg, comparing the sequential use of CR paroxetine after IR paroxetine with continuous use of IR paroxetine). However, we enrolled patients who had residual MDD symptoms after more than 6 months of IR paroxetine use (the mean HSDS score was 12.22 at baseline), making it less likely that symptoms would improve if the patients continued to receive IR therapy. 2) We used patient-rated depression and anxiety scores as the primary effectiveness end point, and although they more accurately reflect the patients' inner mood status, they lack objectivity. 3) We excluded 66 (32.2\%) of 205 subjects who enrolled in this study because of poor adherence (taking less than $80 \%$ of their prescribed CR paroxetine). This was because the primary purpose of this study was to evaluate drug effectiveness and safety in patients who had received a consistent dose for a sufficient duration. The decision to exclude those subjects was arguable because a high attrition rate reduces the power, precision, and generalizability of a study's results. To evaluate the influences of attrition, we conducted a comparison of the included and excluded patients by comparing the results of the per-protocol set and intention-to-treat analysis. In addition, the consistency of the results in both data sets was verified by our findings of no differences in background characteristics (eg, gender or age), rate of discontinuation because of $\mathrm{ADRs}$, and satisfaction with the formulation switch, and by our finding of similar improvements of symptoms and satisfaction (results are shown in Tables S2-S9). Furthermore, the nonadherence rate was consistent with those reported in past clinical studies; antidepressant nonadherence rates in psychiatric populations have been reported to range from $13 \%$ to $55.7 \%,{ }^{22}$ with a 1 -month nonadherence rate of $48.1 \%{ }^{23}$ In future studies, strategies to handle the dropouts should be taken to reduce attrition rates, for example, by adding "poor adherence of prior antidepressants" to primary exclusion criteria, or by providing reimbursement or transportation compensation at each visit. 4) The open nature of the study, with patients aware of the formulation switch, could have led the patients to expect better clinical efficacy. Therefore, the improvements observed in this study could have been caused by psychological effects of changing drugs, including the response of physicians. Thus, to confirm our results, a double-blinded and randomized controlled trial is required.

\section{Conclusion}

Our study results indicate that switching the drug formulation could be a useful option when an initial antidepressant is insufficient to improve the patient's symptoms of depression. Other studies have shown CR paroxetine to be superior to IR paroxetine, based on the findings that it is better tolerated, is more efficacious, and is associated with fewer ADRs, improved symptoms of depression and anxiety, and is associated with lower medical costs. ${ }^{34}$ Satisfaction with paroxetine also improved in approximately $70 \%$ of the patients after changing to the CR treatment. Given these favorable results, switching from IR to CR paroxetine should be considered in clinical practice for patients with MDD who have had a partial response to IR paroxetine or for those suffering from ADRs.

\section{Acknowledgments}

The authors thank Mr. Kenichiro Tsumura for his technical assistance on data analysis, the clinical trial staff at the participating sites for their kind cooperation, and all patients who participated in the trial. The authors would like to thank Enago (www.enago.jp) for the review of English language. Necessary fees for the study, such as analysis of the data, were paid by the Medical Corporation Jisenkai, which runs the Himorogi Psychiatric Institute.

\section{Author contributions}

TO contributed to conception and design, and interpretation of data. YW contributed to conception and design, and acquisition and interpretation of data. SH, MI, KA, KM, and RT contributed to conception, and acquisition and interpretation of data. TK contributed to conception and design, 
and analysis and interpretation of data. TO, YW, and TK drafted this article, and all authors revised it critically. All authors approved the final version of this manuscript and to be published, and agree to be accountable for all aspects of the work.

\section{Disclosure}

TO has received speaker's honoraria from GlaxoSmithKline, Otsuka Pharmaceutical, Eli Lilly Japan, and MSD. YW has received speaker's honoraria from Pfizer, GlaxoSmithKline, Otsuka Pharmaceutical, Janssen Pharmaceutical, Meiji, Eli Lilly Japan, MSD, and Astellas Pharma. SH has received speaker's honoraria from Meiji Seika Pharma, Janssen Pharmaceutical, Eli Lilly Japan, GlaxoSmithKline, Chugai Pharma, Mochida Pharmaceutical, Otsuka Holdings, and Dainippon Sumitomo Pharma. MI has received speaker's honoraria from GlaxoSmithKline, Nippon Zoki Pharmaceutical, AbbVie, and Maruzen. KA has received speaker's honoraria from Astellas Pharma, AbbVie, Asahi Kasei Pharma, and Maruzen. KM has received speaker's honoraria from Eli Lilly Japan, GlaxoSmithKline, Otsuka Pharmaceutical, Janssen Pharmaceutical, Meiji, MSD, Akatsuka, Eisai, Takeda Pharmaceutical, Dainippon Sumitomo Pharma, and Pfizer. RT has received speaker's honoraria from Asahi Kasei Pharma, Astellas Pharma, Otsuka Pharmaceutical, GlaxoSmithKline, Dainippon Sumitomo Pharma, Eli Lilly Japan, Novartis Pharma, Pfizer, Meiji Seika Pharma, Mochida Pharmaceutical, and Janssen Pharmaceutical. TK has received speaker's honoraria from Astellas, Dainippon Sumitomo, Eli Lilly, Janssen, Otsuka, Takeda, Yoshitomi Yakuhin, and Pfizer. The authors report no other conflicts of interest in this work.

\section{References}

1. World Health Organization. Depression. Fact sheet. 2017 [updated February 2017]. Available from: http://www.who.int/mediacentre/factsheets/ fs369/en/. Accessed May 24, 2017.

2. American Psychiatric Association. Practice guideline for the treatment of patients with major depressive disorder. 3rd ed. 2010. Available from: http://psychiatryonline.org/pb/assets/raw/sitewide/practice_guidelines/ guidelines/mdd.pdf. Accessed December 3, 2014.

3. Japanese Society of Mood Disorders. [Treatment guideline II: major depressive disorder, 2013 Ver. 1.1]. 2013. Available from: http:// www.secretariat.ne.jp/jsmd/mood_disorder/img/130924.pdf. Accessed December 3, 2014. Japanese.

4. Rush AJ, Wisniewski SR, Zisook S, et al. Is prior course of illness relevant to acute or longer-term outcomes in depressed out-patients? A STAR*D report. Psychol Med. 2012;42(6):1131-1149.

5. Bauer M, Bschor T, Pfennig A, et al; WFSBP Task Force on Unipolar Depressive Disorders. World Federation of Societies of Biological Psychiatry (WFSBP) guidelines for biological treatment of unipolar depressive disorders in primary care. World J Biol Psychiatry. 2007;8(2):67-104.
6. Anderson IM, Ferrier IN, Baldwin RC, et al. Evidence-based guidelines for treating depressive disorders with antidepressants: a revision of the British Association for Psychopharmacology guidelines. J Psychopharmacol. 2008;22(4):343-396.

7. Papakostas GI, Fava M, Thase ME. Treatment of SSRI-resistant depression: a meta-analysis comparing within- versus across-class switches. Biol Psychiatry. 2008;63(7):699-704.

8. Guico-Pabia CJ, Jiang Q, Ninan PT, Thase ME. Clinical outcomes following switch from venlafaxine ER to desvenlafaxine in nonresponders and responders. Curr Med Res Opin. 2011;27(9):1815-1826.

9. Higuchi T, Hong JP, Jung HY, Watanabe Y, Kunitomi T, Kamijima K. Paroxetine controlled-release formulation in the treatment of major depressive disorder: a randomized, double-blind, placebo-controlled study in Japan and Korea. Psychiatry Clin Neurosci. 2011;65(7): 655-663.

10. Golden RN, Nemeroff CB, McSorley P, Pitts CD, Dubé EM. Efficacy and tolerability of controlled-release and immediate-release paroxetine in the treatment of depression. J Clin Psychiatry. 2002; 63(7):577-584.

11. Kato M, Kimura T, Kimura T, Hara T. Safety and effectiveness of controlled-release paroxetine in routine clinical practice: results of a postmarketing surveillance study of patients with depression. Neuropsychiatr Dis Treat. 2015;11:435-452.

12. DeVane CL. Pharmacokinetics, drug interactions, and tolerability of paroxetine and paroxetine CR. Psychopharmacol Bull. 2003;37 (Suppl 1): $29-41$.

13. Golden RN. Efficacy and tolerability of controlled-release paroxetine. Psychopharmacol Bull. 2003;37 (Suppl 1):176-186.

14. Ramsberg J, Asseburg C, Henriksson M. Effectiveness and costeffectiveness of antidepressants in primary care: a multiple treatment comparison meta-analysis and cost-effectiveness model. PLoS One. 2012; 7(8): 42003.

15. Kishi T, Matsuda $Y$, Matsunaga S, et al. Escitalopram versus paroxetine controlled release in major depressive disorder: a randomized trial. Neuropsychiatr Dis Treat. 2017;13:117-125.

16. Mimura C, Murashige M, Oda T, Watanabe Y. Development and psychometric evaluation of a Japanese scale to assess depression severity: Himorogi self-rating depression scale. Int J Psychiatry Clin Pract. 2011; 15(1):50-55.

17. Mimura C, Nishioka M, Sato N, Hasegawa R, Horikoshi R, Watanabe Y. A Japanese scale to assess anxiety severity: development and psychometric evaluation. Int J Psychiatry Med. 2011;41(1):29-45.

18. Hamilton M. Development of a rating scale for primary depressive illness. Br J Soc Clin Psychol. 1967;6(4):278-296.

19. Bruss GS, Gruenberg AM, Goldstein RD, Barber JP. Hamilton Anxiety Rating Scale Interview guide: joint interview and testretest methods for interrater reliability. Psychiatry Res. 1994;53(2): 191-202.

20. Guy W. ECDEU assessment manual for psychopharmacology: publication ADM 76-338. Clinical Global Impressions (CGI) Scale. Washington, DC: Department of Health, Education, and Welfare; 1976:218-222.

21. Busner J, Targum SD. The clinical global impressions scale: applying a research tool in clinical practice. Psychiatry (Edgmont). 2007;4(7): $28-37$.

22. Sansone RA, Sansone LA. SSRIs: bad to the bone? Innov Clin Neurosci. 2012;9(7-8):42-47.

23. Lee MS, Lee HY, Kang SG, et al. Variables influencing antidepressant medication adherence for treating outpatients with depressive disorders. J Affect Disord. 2010;123(1-3):216-221.

24. Jakubovski E, Varignonda AL, Freemantle N, Taylor MJ, Bloch MH. Systematic review and meta-analysis: dose-response relationship of selective serotonin reuptake inhibitors in major depressive disorder. Am J Psychiatry. 2016;173(2):173-183.

25. Patel MX, Arista IA, Taylor M, Barnes TR. How to compare doses of different antipsychotics: a systematic review of methods. Schizophr Res. 2013;149(1-3):141-148. 
26. Hayasaka Y, Purgato M, Magni LR, et al. Dose equivalents of antidepressants: evidence-based recommendations from randomized controlled trials. J Affect Disord. 2015;180:179-184.

27. Bschor T, Kern H, Henssler J, Baethge C. Switching the antidepressant after nonresponse in adults with major depression: a systematic literature search and meta-analysis. J Clin Psychiatry. Epub 2016 Dec 6.

28. Lin EH, Von Korff M, Katon W, et al. The role of the primary care physician in patients' adherence to antidepressant therapy. Med Care. 1995;33(1):67-74.

29. Bull SA, Hu XH, Hunkeler EM, et al. Discontinuation of use and switching of antidepressants: influence of patient-physician communication. JAMA. 2002;288(11):1403-1409.

30. Keene MS, Eaddy MT, Nelson WW, Sarnes MW. Adherence to paroxetine CR compared with paroxetine IR in a Medicare-eligible population with anxiety disorders. Am J Manag Care. 2005;11(12 Suppl): S362-S369.
31. Aljumah K, Ahmad Hassali A, AlQhatani S. Examining the relationship between adherence and satisfaction with antidepressant treatment. Neuropsychiatr Dis Treat. 2014;10:1433-1438.

32. Sivertsen H, Bjørkløf GH, Engedal K, Selbæk G, Helvik AS. Depression and quality of life in older persons: a review. Dement Geriatr Cogn Disord. 2015;40(5-6):311-339.

33. Fava M, Rush AJ, Alpert JE, et al. Difference in treatment outcome in outpatients with anxious versus nonanxious depression: a STAR*D report. Am J Psychiatry. 2008;165(3):342-351.

34. Sheehan DV, Eaddy MT, Shah MB, Mauch RP. Differences in total medical costs across the SSRIs for the treatment of depression and anxiety. Am J Manag Care. 2005;11(12 Suppl):S354-S361.

35. Pharmaceuticals and Medical Devices Agency. [Drug information of paroxetine CR $12.5 \mathrm{mg} / 25 \mathrm{mg}$ ]. Available from: http://www.info.pmda. go.jp/go/pack/1179041G1020_1_08/. Accessed December 24, 2017. Japanese. 


\section{Supplementary materials}

Table SI Shift of HSDS scores from week 0 to week 8 in PPS $(n=1 \mid 3)$

\begin{tabular}{|c|c|c|c|c|c|c|}
\hline \multirow[t]{2}{*}{ Scores } & \multicolumn{6}{|c|}{ HSDS (OW) } \\
\hline & 0-9 & $10-15$ & $16-20$ & $21-30$ & $\geq 31$ & Total \\
\hline \multicolumn{7}{|c|}{ HSDS (8W) } \\
\hline $0-9$ & 47 & 9 & 2 & 0 & I & 59 \\
\hline $10-15$ & 4 & 12 & 9 & 2 & I & 28 \\
\hline $16-20$ & 1 & I & 10 & 3 & 0 & 15 \\
\hline $21-30$ & 0 & 0 & I & 9 & 0 & 10 \\
\hline$\geq 31$ & 0 & 0 & 0 & I & 0 & I \\
\hline Total & 52 & 22 & 22 & 15 & 2 & 113 \\
\hline
\end{tabular}

Notes: HSDS scores: 0-9, no depression; 10-15, mild depression; 16-20, moderate depression; $2 \mathrm{I}-30$, moderately severe depression; and $\geq 3 \mathrm{I}$, severe depression.

Abbreviations: HSDS, Himorogi Self-Rating Depression Scale; PPS, per-protocol set.

Table S2 Baseline characteristics

\begin{tabular}{|c|c|c|c|}
\hline Characteristics & PPS, $n=1$ I 3 & ITT, n=205 & $P$-value \\
\hline \multicolumn{4}{|l|}{ Gender, n (\%) } \\
\hline Female & $57(50.4)$ & $110(53.7)$ & $0.639 @$ \\
\hline Age, mean \pm SD (years) & $41.2 \pm 11.52$ & $41.4 \pm 11.28$ & $0.898^{\ddagger}$ \\
\hline \multicolumn{4}{|l|}{ Diagnosis, n (\%) } \\
\hline MDD & $77(68.1)$ & |46 (7I.2) & $0.983^{\pi}$ \\
\hline MDD with SAD & $22(19.5)$ & $35(17.1)$ & \\
\hline MDD with PD & $9(8.0)$ & $16(7.8)$ & \\
\hline MDD with OCD & $2(1.8)$ & $3(1.5)$ & \\
\hline MDD with other comorbidities & $3(2.7)$ & $5(2.4)$ & \\
\hline \multicolumn{4}{|l|}{ CR paroxetine initial dose, $n(\%)$} \\
\hline $12.5 \mathrm{mg}$ & $31(27.4)$ & $67(32.7)$ & $0.365^{\S}$ \\
\hline $25 \mathrm{mg}$ & $37(32.7)$ & $63(30.7)$ & \\
\hline $37.5 \mathrm{mg}$ & $15(13.3)$ & $27(13.2)$ & \\
\hline $50 \mathrm{mg}$ & $30(26.5)$ & $48(23.4)$ & \\
\hline
\end{tabular}

Notes: ${ }^{\circledR}$ Fisher's exact test. ${ }^{\ddagger}$ Student's $t$-test. "Chi-square test. ${ }^{\circledR}$ Mann-Whitney $U$ test. Abbreviations: PPS, per-protocol set; ITT, intention to treat; SD, standard deviation; MDD, major depressive disorder; SAD, social anxiety disorder; PD, panic disorder; OCD, obsessive-compulsive disorder; $C R$, controlled release.
Table S3 Baseline characteristics

\begin{tabular}{|c|c|c|c|}
\hline Characteristics & PPS, $n=113$ & ITT, $\mathrm{n}=\mathbf{2 0 5}$ & $P$-value \\
\hline \multicolumn{4}{|l|}{ CGI-S, n (\%) } \\
\hline Normal & $8(7.1)$ & $17(8.3)$ & $0.282^{\S}$ \\
\hline Borderline medically ill & $30(26.5)$ & $39(19.0)$ & \\
\hline Mildly ill & $52(46.0)$ & $91(44.4)$ & \\
\hline Moderately ill & $20(17.7)$ & $45(22.0)$ & \\
\hline Markedly ill & $3(2.7)$ & $6(2.9)$ & \\
\hline Severely ill & $0(0.0)$ & $0(0.0)$ & \\
\hline Unknown & $0(0.0)$ & $7(3.4)$ & \\
\hline \multicolumn{4}{|c|}{ Satisfaction and preference for paroxetine (SSMQ), $n(\%)$} \\
\hline Dissatisfied & $4(3.5)$ & $9(4.4)$ & $\left.0.80\right|^{\S}$ \\
\hline Slightly dissatisfied & $26(23.1)$ & $40(19.5)$ & \\
\hline Neutral & $23(20.4)$ & $46(22.3)$ & \\
\hline Slightly satisfied & $25(22.1)$ & $44(2 I .5)$ & \\
\hline Satisfied & $30(26.5)$ & $42(20.5)$ & \\
\hline Very satisfied & $5(4.4)$ & $12(5.9)$ & \\
\hline Unknown & $0(0.0)$ & $12(5.9)$ & \\
\hline \multicolumn{4}{|c|}{ Patient preference for CR paroxetine (MPQ), $n(\%)$} \\
\hline Much better & $6(5.3)$ & $14(6.8)$ & $0.737^{\S}$ \\
\hline Moderately better & $32(28.3)$ & $48(23.4)$ & \\
\hline Slightly better & $19(16.8)$ & $32(15.6)$ & \\
\hline Similar & $29(25.7)$ & $52(25.4)$ & \\
\hline Slightly worse & $22(19.5)$ & $36(17.6)$ & \\
\hline Worse & $5(4.4)$ & $13(6.3)$ & \\
\hline Unknown & $0(0.0)$ & $10(4.9)$ & \\
\hline
\end{tabular}

Note: \$Mann-Whitney $U$ test.

Abbreviations: PPS, per-protocol set; ITT, intention to treat; CGI-S, Clinical Global Impression - Severity; SSMQ, Satisfaction with Study Medication Question; $\mathrm{CR}$, controlled release; MPQ, Medication Preference Question. 
Table S4 Depression/anxiety scores at baseline

\begin{tabular}{|c|c|c|c|}
\hline Characteristics & PPS, $n=I \mid 3$ & ITT, $@ n=20$ & $P$-value \\
\hline \multicolumn{4}{|l|}{ HSDS score (week 0 ), mean \pm SD } \\
\hline Total score & $12.22 \pm 8.16$ & $13.60 \pm 8.83$ & 0.172 \\
\hline I. Sleep disturbance & $2.11 \pm 2.40$ & $2.25 \pm 2.44 \S$ & 0.613 \\
\hline 2. Appetite & $0.65 \pm 1.12$ & $0.77 \pm 1.18$ & $<0.00 I^{* *}$ \\
\hline 3. Anxiety & $1.98 \pm 1.70$ & $2.22 \pm 1.76$ & 0.248 \\
\hline 4. Fatigue & $1.36 \pm 0.94$ & $\mathrm{I} .5 \mathrm{I} \pm 0.95$ & 0.186 \\
\hline 5. Hyposexuality & $1.30 \pm 0.99$ & $1.39 \pm 1.03$ & 0.481 \\
\hline 6. Somatic disorder & $1.10 \pm 0.91$ & $1.25 \pm 0.95$ & 0.169 \\
\hline 7. Depression & $0.7 I \pm 0.73^{@}$ & $0.76 \pm 0.73^{\S}$ & 0.567 \\
\hline 8. Disinclination & $1.27 \pm 0.90$ & $1.31 \pm 0.91$ & 0.742 \\
\hline 9. Diminished ability to think & $0.85 \pm 0.83$ & $0.94 \pm 0.85$ & 0.365 \\
\hline 10. Lower satisfaction & $0.87 \pm 0.88 @$ & $0.96 \pm 0.93 \pi$ & 0.401 \\
\hline \multicolumn{4}{|l|}{ HSAS score (week 0 ), mean \pm SD } \\
\hline Total score & $12.72 \pm 9.53$ & $13.95 \pm 9.77$ & 0.281 \\
\hline I. Cardiovascular & $\mid .5 \mathrm{I} \pm \mathrm{I} .79$ & $1.66 \pm 1.79$ & 0.406 \\
\hline 2. Respiratory & $1.86 \pm 1.94$ & $2.07 \pm 2.02$ & 0.386 \\
\hline 3. Autonomic nerve & $2.7 I \pm 2.13$ & $3.02 \pm 2.20 \S$ & 0.246 \\
\hline 4. Gastrointestinal & $1.13 \pm 1.00 @$ & $1.19 \pm 1.03^{\S}$ & 0.682 \\
\hline 5. Distress & $1.27 \pm 1.00^{\ddagger}$ & $1.39 \pm 1.04$ & 0.304 \\
\hline 6. Awful feeling & $0.66 \pm 0.93$ & $0.73 \pm 0.94$ & 0.509 \\
\hline 7. Anticipatory anxiety & $\mathrm{I} .0 \mathrm{I} \pm \mathrm{I} .0 \mathrm{I}$ & $1.06 \pm 1.03$ & 0.719 \\
\hline 8. Sleeping & $1.10 \pm 1.09$ & $1.22 \pm 1.13$ & 0.347 \\
\hline 9. Depersonalization & $0.50 \pm 0.86$ & $0.61 \pm 0.90$ & 0.172 \\
\hline 10. Anxiousness & $1.00 \pm 0.97$ & $1.02 \pm 1.01$ & 0.925 \\
\hline
\end{tabular}

Notes: There were four subjects with missing data at baseline in ITT $(n=205)$ group. Evaluations were conducted at baseline between PPS $(n=113)$ and ITT $(n=205)$ groups. HSDS scores: 0-9, no depression; 10-15, mild depression; 16-20, moderate depression; 21-30, moderately severe depression; and $\geq 31$, severe depression. HSAS scores: 0-7, no anxiety; 8-15, mild anxiety; 16-20, moderate anxiety; 21-30, moderately severe anxiety; and $\geq 31$, severe anxiety. ${ }^{{ }} n=112$. ${ }^{\ddagger} n=11 I .{ }^{5} n=200$. $\pi_{n=199 .} * *$ Statistically significant at $\alpha=0.01$ by Student's $t$-test (non-paired).

Abbreviations: PPS, per-protocol set; ITT, intention to treat; HSDS, Himorogi Self-Rating Depression Scale; SD, standard deviation; HSAS, Himorogi Self-Rating Anxiety Scale.
Table S5 Depression/anxiety scores at week 8

\begin{tabular}{|c|c|c|c|}
\hline Characteristics & PPS, $n=I \mid 3$ & ITT, $n=153$ & $P$-value \\
\hline \multicolumn{4}{|l|}{ HSDS score (week 8), mean \pm SD } \\
\hline Total score & $10.22 \pm 7.24$ & $11.90 \pm 7.83$ & 0.075 \\
\hline I. Sleep disturbance & $1.65 \pm 2.19$ & $1.93 \pm 2.30$ & 0.289 \\
\hline 2. Appetite & $0.48 \pm 0.98 @$ & $0.63 \pm 1.04 \S$ & 0.185 \\
\hline 3. Anxiety & $1.70 \pm 1.6 \mathrm{I}$ & $2.07 \pm 1.77$ & 0.098 \\
\hline 4. Fatigue & $1.22 \pm 0.90$ & $1.36 \pm 0.94$ & 0.222 \\
\hline 5. Hyposexuality & $1.05 \pm 0.96$ & $\mathrm{I} .1 \mathrm{I} \pm 0.96$ & 0.591 \\
\hline 6. Somatic disorder & $1.00 \pm 0.88$ & $1.15 \pm 0.94$ & 0.203 \\
\hline 7. Depression & $0.63 \pm 0.73$ & $0.75 \pm 0.76$ & $0.16 \mathrm{I}$ \\
\hline 8. Disinclination & $1.09 \pm 0.87$ & $1.23 \pm 0.89$ & $0.66 \mathrm{I}$ \\
\hline 9. Diminished ability to think & $0.73 \pm 0.74$ & $0.84 \pm 0.76^{\S}$ & 0.254 \\
\hline 10. Lower satisfaction & $0.68 \pm 0.77$ & $0.85 \pm 0.85$ & 0.106 \\
\hline \multicolumn{4}{|l|}{ HSAS score (week 8), mean \pm SD } \\
\hline Total score & $11.18 \pm 8.86$ & $12.69 \pm 9.36$ & 0.185 \\
\hline I. Cardiovascular & $1.29 \pm 1.62$ & $1.49 \pm 1.77$ & 0.414 \\
\hline 2. Respiratory & $1.68 \pm 1.86$ & $1.99 \pm 1.98$ & 0.212 \\
\hline 3. Autonomic nerve & $2.42 \pm 1.92$ & $2.67 \pm 2.03$ & 0.342 \\
\hline 4. Gastrointestinal & $1.1 \mathrm{I} \pm 1.00$ & $1.19 \pm 1.03$ & 0.529 \\
\hline 5. Distress & $1.11 \pm 0.98$ & $1.23 \pm 0.98$ & 0.283 \\
\hline 6. Awful feeling & $0.55 \pm 0.91$ & $0.63 \pm 0.93$ & 0.416 \\
\hline 7. Anticipatory anxiety & $0.82 \pm 0.90$ & $0.92 \pm 0.91$ & 0.334 \\
\hline 8. Sleeping & $0.88 \pm 1.01$ & $1.02 \pm 1.05 \pi$ & 0.259 \\
\hline 9. Depersonalization & $0.42 \pm 0.79$ & $0.55 \pm 0.88$ & 0.268 \\
\hline 10. Anxiousness & $0.92 \pm 1.01 @$ & $1.03 \pm 1.03^{\S}$ & 0.388 \\
\hline
\end{tabular}

Notes: There were 52 dropouts at week 8 in ITT $(n=205)$ group. Evaluations were conducted at week 8 between PPS $(n=\mid 13)$ and ITT $(n=153)$ groups. HSDS scores: 0-9, no depression; 10-15, mild depression; 16-20, moderate depression; 21-30, moderately severe depression; and $\geq 3 \mathrm{I}$, severe depression. HSAS scores: $0-7$, no anxiety; 8-15, mild anxiety; 16-20, moderate anxiety; 21-30, moderately severe anxiety; and $\geq 31$, severe anxiety. ${ }^{{ }} n=\mid 12$. ${ }^{n} n=111$. ${ }^{8} n=\mid 52$. ${ }^{\pi n} n=|5|$.

Abbreviations: PPS, per-protocol set; ITT, intention to treat; HSDS, Himorogi Self-Rating Depression Scale; SD, standard deviation; HSAS, Himorogi Self-Rating Anxiety Scale. 
Table S6 Changes in depression/anxiety scores in ITT $(n=\mid 50)$

\begin{tabular}{|c|c|c|c|}
\hline Scale items & Baseline & Week 8 & $P$-value \\
\hline \multicolumn{4}{|l|}{ HSDS score, mean \pm SD } \\
\hline Total score & $13.39 \pm 8.60$ & 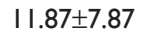 & $0.002 * *$ \\
\hline I. Sleep disturbance & $2.25 \pm 2.46$ & $1.95 \pm 2.32$ & 0.080 \\
\hline 2. Appetite & $0.77 \pm 1.22$ & $0.64 \pm 1.05$ & 0.235 \\
\hline 3. Anxiety & $2.19 \pm 1.78$ & $2.04 \pm 1.77 @$ & 0.259 \\
\hline 4. Fatigue & $1.47 \pm 0.96$ & $1.37 \pm 0.89$ & 0.148 \\
\hline 5. Hyposexuality & $1.33 \pm 1.01$ & $1.10 \pm 0.97$ & $<0.00 I^{* *}$ \\
\hline 6. Somatic disorder & $1.20 \pm 0.93$ & $1.15 \pm 0.94$ & 0.373 \\
\hline 7. Depression & $0.78 \pm 0.77$ & $0.74 \pm 0.77$ & 0.462 \\
\hline 8. Disinclination & $1.35 \pm 0.92$ & $1.23 \pm 0.90$ & 0.075 \\
\hline 9. Diminished ability to think & $0.97 \pm 0.90$ & $0.83 \pm 0.77$ & $0.020^{*}$ \\
\hline 10. Lower satisfaction & $0.99 \pm 0.95$ & $0.85 \pm 0.86$ & $0.019 *$ \\
\hline \multicolumn{4}{|l|}{ HSAS score, mean \pm SD } \\
\hline Total score & $13.76 \pm 9.72$ & $12.35 \pm 9.28$ & $0.002 * *$ \\
\hline I. Cardiovascular & $1.65 \pm 1.81$ & I.47士I.78@ & 0.141 \\
\hline 2. Respiratory & $2.09 \pm 2.03$ & $1.96 \pm 1.99$ & 0.309 \\
\hline 3. Autonomic nerve & $2.92 \pm 2.17$ & $2.69 \pm 2.04$ & 0.071 \\
\hline 4. Gastrointestinal & $1.24 \pm 1.03$ & 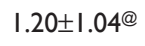 & 0.630 \\
\hline 5. Distress & $1.42 \pm 1.04$ & $1.22 \pm 0.98^{\ddagger}$ & $0.004 * *$ \\
\hline 6. Awful feeling & $0.75 \pm 0.98$ & $0.63 \pm 0.93$ & $0.029 *$ \\
\hline 7. Anticipatory anxiety & $1.07 \pm 1.02$ & $0.92 \pm 0.92 @$ & $0.019 *$ \\
\hline 8. Sleeping & $1.24 \pm 1.13$ & $1.02 \pm 1.07$ & $0.007 * *$ \\
\hline 9. Depersonalization & $0.63 \pm 0.93$ & $0.54 \pm 0.89 @$ & 0.091 \\
\hline 10. Anxiousness & $1.04 \pm 1.01$ & I.02土1.04@ & 0.764 \\
\hline
\end{tabular}

Notes: Primary comparisons were made between baseline and week 8 scores in ITT group. Data from week 4 were not included in the final analyses. HSDS scores: 0-9, no depression; 10-15, mild depression; 16-20, moderate depression; 21-30, moderately severe depression; and $\geq 31$, severe depression. HSAS scores: $0-7$, no anxiety; 8-15, mild anxiety; 16-20, moderate anxiety; 21-30, moderately severe anxiety; and $\geq 31$, severe anxiety. ${ }^{@} \mathrm{n}=149 .{ }^{{ }^{n}} \mathrm{n}=148$. $*$ Statistically significant at $\alpha=0.05$ **Statistically significant at $\alpha=0.0$ I by Student's paired $t$-test.

Abbreviations: ITT, intention to treat; HSDS, Himorogi Self-Rating Depression Scale; SD, standard deviation; HSAS, Himorogi Self-Rating Anxiety Scale.
Table S7 CGI-S, SSMQ, and MPQ scores at baseline

\begin{tabular}{|c|c|c|c|}
\hline Characteristics & PPS, $n=I \mid 3$ & ITT, $\mathbf{n = 2 0 5}$ & $P$-value \\
\hline \multicolumn{4}{|l|}{ CGI-S, n (\%) } \\
\hline I. Normal & $8(7.1)$ & $17(8.3)$ & 0.282 \\
\hline 2. Borderline mentally ill & $30(26.5)$ & $39(19.0)$ & \\
\hline 3. Mildly ill & $52(46.0)$ & $91(44.4)$ & \\
\hline 4. Moderately ill & $20(17.7)$ & $45(22.0)$ & \\
\hline 5. Markedly ill & $3(2.7)$ & $6(2.9)$ & \\
\hline 6. Severely ill & $0(0.0)$ & $0(0.0)$ & \\
\hline Unknown & $0(0.0)$ & $7(3.4)$ & \\
\hline \multicolumn{4}{|l|}{ SSMQ, n (\%) } \\
\hline I. Dissatisfied & $4(3.5)$ & $9(4.4)$ & 0.801 \\
\hline 2. Slightly dissatisfied & $26(23.0)$ & $40(19.5)$ & \\
\hline 3. Neutral & $23(20.4)$ & $46(22.4)$ & \\
\hline 4. Slightly satisfied & $25(22.1)$ & $44(2 \mid .4)$ & \\
\hline 5. Satisfied & $30(26.5)$ & $42(20.5)$ & \\
\hline 6. Very satisfied & $5(4.4)$ & $12(5.9)$ & \\
\hline Unknown & $0(0.0)$ & $12(5.9)$ & \\
\hline \multicolumn{4}{|l|}{ MPQ, n (\%) } \\
\hline I. Much better & $6(5.3)$ & $14(6.8)$ & 0.737 \\
\hline 2. Moderately better & $32(28.3)$ & $48(23.4)$ & \\
\hline 3. Slightly better & $19(16.8)$ & $32(15.6)$ & \\
\hline 4. Similar & $29(25.7)$ & $52(25.4)$ & \\
\hline 5. Slightly worse & $22(19.5)$ & $36(17.6)$ & \\
\hline 6. Worse & $5(4.4)$ & $13(6.3)$ & \\
\hline Unknown & $0(0.0)$ & $10(4.9)$ & \\
\hline
\end{tabular}

Notes: Evaluations were conducted at baseline between PPS $(n=\mid 13)$ and ITT $(n=205)$ groups.

Abbreviations: CGI-S, Clinical Global Impression - Severity; SSMQ, Satisfaction with Study Medication Question; MPQ, Medication Preference Question; PPS, perprotocol set; ITT, intention to treat. 
Table $\mathbf{S 8}$ CGI-S, SSMQ, and MPQ scores at week 8

\begin{tabular}{|c|c|c|c|}
\hline Characteristics & PPS, $n=113$ & ITT, $n=I 54$ & $P$-value \\
\hline \multicolumn{4}{|l|}{ CGI-S, n (\%) } \\
\hline I. Normal & $10(8.8)$ & $14(9.1)$ & 0.179 \\
\hline 2. Borderline mentally ill & $50(44.2)$ & $53(34.4)$ & \\
\hline 3. Mildly ill & $40(35.4)$ & $65(42.2)$ & \\
\hline 4. Moderately ill & $13(11.5)$ & $19(12.3)$ & \\
\hline 5. Markedly ill & $0(0.0)$ & $3(1.9)$ & \\
\hline 6. Severely ill & $0(0.0)$ & $0(0.0)$ & \\
\hline Unknown & $0(0.0)$ & $0(0.0)$ & \\
\hline \multicolumn{4}{|l|}{ SSMQ, n (\%) } \\
\hline I. Dissatisfied & $0(0.0)$ & I (0.6) & 0.482 \\
\hline 2. Slightly dissatisfied & I (0.9) & $2(1.3)$ & \\
\hline 3. Neutral & $31(27.4)$ & $44(28.6)$ & \\
\hline 4. Slightly satisfied & $20(17.7)$ & $32(20.8)$ & \\
\hline 5. Satisfied & $47(41.6)$ & $57(37.0)$ & \\
\hline 6. Very satisfied & $14(12.4)$ & 18 (11.7) & \\
\hline Unknown & $0(0.0)$ & $0(0.0)$ & \\
\hline \multicolumn{4}{|l|}{ MPQ, n (\%) } \\
\hline I. Much better & $16(14.2)$ & $22(14.3)$ & 0.781 \\
\hline 2. Moderately better & $44(38.9)$ & $55(35.7)$ & \\
\hline 3. Slightly better & $15(13.3)$ & $25(16.2)$ & \\
\hline 4. Similar & $36(31.9)$ & $48(31.2)$ & \\
\hline 5. Slightly worse & $2(1.8)$ & $3(1.9)$ & \\
\hline 6. Worse & $0(0.0)$ & I (0.6) & \\
\hline Unknown & $0(0.0)$ & $0(0.0)$ & \\
\hline
\end{tabular}

Notes: There were 5 I dropouts at week 8 in ITT $(n=205)$ group. Evaluations were conducted at week 8 between PPS $(n=\mid 13)$ and ITT $(n=154)$ groups.

Abbreviations: CGI-S, Clinical Global Impression - Severity; SSMQ, Satisfaction with Study Medication Question; MPQ, Medication Preference Question; PPS, perprotocol set; ITT, intention to treat.
Table S9 Changes of CGI-S, SSMQ, and MPQ scores in ITT $(n=151)$ group

\begin{tabular}{|c|c|c|c|}
\hline Characteristics & Week 0 & Week 8 & $P$-value \\
\hline \multicolumn{4}{|l|}{ CGI-S, n (\%) } \\
\hline I. Normal & $10(6.7)$ & $14(9.3)$ & $<0.00 I^{* *}$ \\
\hline 2. Borderline mentally ill & 31 (20.7) & $53(35.3)$ & \\
\hline 3. Mildly ill & $71(47.3)$ & $63(42.0)$ & \\
\hline 4. Moderately ill & $36(24.0)$ & $18(12.0)$ & \\
\hline 5. Markedly ill & $2(1.3)$ & $2(1.3)$ & \\
\hline 6. Severely ill & $0(0.0)$ & $0(0.0)$ & \\
\hline \multicolumn{4}{|l|}{ SSMQ, n (\%) } \\
\hline I. Dissatisfied & $7(4.6)$ & I (0.7) & $<0.00 I^{* *}$ \\
\hline 2. Slightly dissatisfied & $35(23.2)$ & $2(1.3)$ & \\
\hline 3. Neutral & $36(23.8)$ & $44(29.1)$ & \\
\hline 4. Slightly satisfied & $35(23.2)$ & $29(19.2)$ & \\
\hline 5. Satisfied & $32(21.2)$ & $57(37.7)$ & \\
\hline 6. Very satisfied & $6(4.0)$ & $18(11.9)$ & \\
\hline \multicolumn{4}{|l|}{ MPQ, n (\%) } \\
\hline I. Much better & $8(5.3)$ & $22(14.6)$ & $<0.00 I^{* *}$ \\
\hline 2. Moderately better & $36(23.8)$ & $55(36.4)$ & \\
\hline 3. Slightly better & $23(15.2)$ & $22(14.6)$ & \\
\hline 4. Similar & $43(28.5)$ & $48(31.8)$ & \\
\hline 5. Slightly worse & $31(20.5)$ & $3(2.0)$ & \\
\hline 6. Worse & $10(6.6)$ & I (0.7) & \\
\hline Unknown & $0(0.0)$ & $0(0.0)$ & \\
\hline
\end{tabular}

Notes: $\mathrm{A}$ total of $\mathrm{I} 50$ subjects were evaluated using CGI-S. Evaluations were conducted in ITT group between week 0 and week 8 . **Statistically significant at $\alpha=0.0$ l by Wilcoxon signed rank-sum test.

Abbreviations: CGI-S, Clinical Global Impression - Severity; SSMQ, Satisfaction with Study Medication Question; MPQ, Medication Preference Question; ITT, intention to treat.
Neuropsychiatric Disease and Treatment

\section{Publish your work in this journal}

Neuropsychiatric Disease and Treatment is an international, peerreviewed journal of clinical therapeutics and pharmacology focusing on concise rapid reporting of clinical or pre-clinical studies on a range of neuropsychiatric and neurological disorders. This journal is indexed on PubMed Central, the 'PsycINFO' database and CAS,

\section{Dovepress}

and is the official journal of The International Neuropsychiatric Association (INA). The manuscript management system is completely online and includes a very quick and fair peer-review system, which is all easy to use. Visit http://www.dovepress.com/testimonials.php to read real quotes from published authors. 